\title{
Helium variation due to self-pollution among Globular Cluster stars
}

\section{Consequences on the horizontal branch morphology}

\author{
F. D’Antona ${ }^{1}$, V. Caloi ${ }^{2}$, J. Montalbán $^{1}$, P. Ventura ${ }^{1}$, and R. Gratton ${ }^{3}$ \\ 1 INAF, Osservatorio Astronomico di Roma, via di Frascati 33, 00040 Monteporzio, Italy \\ 2 Istituto di Astrofisica Spaziale e Fisica Cosmica CNR, via Fosso del Cavaliere, 00133 Roma, Italy \\ e-mail: caloi@rm.iasf.cnr.it \\ 3 INAF, Osservatorio Astronomico di Padova, vicolo dell' Osservatorio, 5, 35122 Padova, Italy \\ e-mail: gratton@mostro.pd.astro.it
}

Received 19 June 2002 / Accepted 23 August 2002

\begin{abstract}
It is becoming clear that "self-pollution" by the ejecta of massive asymptotic giant branch stars has an important role in the early chemical evolution of globular cluster stars, producing CNO abundance spreads which are observed also at the surface of unevolved stars. Observing that the ejecta which are CNO processed must also be helium enriched, we have modelled stellar evolution of globular cluster stars by taking into account this possible helium enhancement with respect to the primordial value. We show that the differences between the main evolutionary phases (main sequence, turn-off and red giants) are small enough that it would be very difficult to detect them observationally. However, the difference in the evolving mass may play a role in the morphology of the horizontal branch, and in particular in the formation of blue tails, in those globular clusters which show strong CNO abundance variations, such as M13 and NGC 6752.
\end{abstract}

Key words. stars: abundances - stars: horizontal branch - globular clusters: general

\section{Introduction}

The chemical inhomogeneities (spread in the abundances of $\mathrm{CNO}, \mathrm{O}-\mathrm{Na}$ and $\mathrm{Mg}-\mathrm{Al}$ anticorrelation) observed in globular cluster (GC) members from the main sequence to the red giant branch impinge on problems such as cluster formation and early evolution (see, e.g., Kraft 1994; Gratton et al. 2001). In some clusters the peculiarities are particularly wide spread and strong, as in M 13 and NGC 6752 (Da Costa \& Cottrell 1980; Norris et al. 1981; Smith \& Norris 1993; Pilachowski et al. 1996; Kraft et al. 1997; Sneden 2000; Gratton et al. 2001). The fact that these clusters present also the "anomaly" of an extended and exclusively blue horizontal branch has been noticed by Catelan \& de Freitas Pacheco (1995), who observe that clusters with super oxygen-poor giants have all very blue HBs. On the other hand, it seems most likely that there is not a direct relation between the two features (i.e., blue tail and anomalies), but rather that they may share the same origin, at least in part.

The most common hypotheses for the origin of the chemical peculiarities are either deep mixing in the cluster members of material nuclearly processed in their interior, or pollution partial or total - by external material (see, e.g., Bell et al. 1981;

Send offprint requests to: F. D’Antona, e-mail: dantona@coma.mporzio.astro.it
Cottrell \& Da Costa 1981; D’Antona et al. 1983; Kraft 1994; Cannon et al. 1998). The confirmation that turn-off stars show peculiarities quite similar to those observed in giants casts severe doubts on the deep mixing scenario (Gratton et al. 2001).

The primordial pollution hypothesis requires either contamination of intracluster material, out of which new stars form with a chemical composition different from a preceding star generation, or partial contamination (superficial or deep) of pre-existing main sequence structures. The contaminating objects are generally identified with intermediate mass asymptotic giant branch (AGB) stars (4-6 $M_{\odot}$ stars, see D'Antona et al. 1983; Ventura et al. 2001, 2002; Thoul et al. 2002), which evolve rapidly enough to shed their envelopes in about $10^{8} \mathrm{yr}$.

The detailed composition of these envelopes is not easy to predict, as it depends on the uncertain physics of convection and mass loss in the intermediate mass AGB stars, but it will surely show an enrichment in helium, in part as a consequence of the second dredge-up, and in part due to the third dredge-up: the helium mass fraction should be $\gtrsim 0.29$ instead of $\sim 0.24$, the cosmic value (Ventura et al. 2001) ${ }^{1}$. When these envelopes are

${ }^{1}$ Notice that the value $Y=0.29$ for the ejecta of the most massive AGBs is substantially conservative, as the Ventura et al. models make very conservative assumptions on the modalities of the third dredge up. 


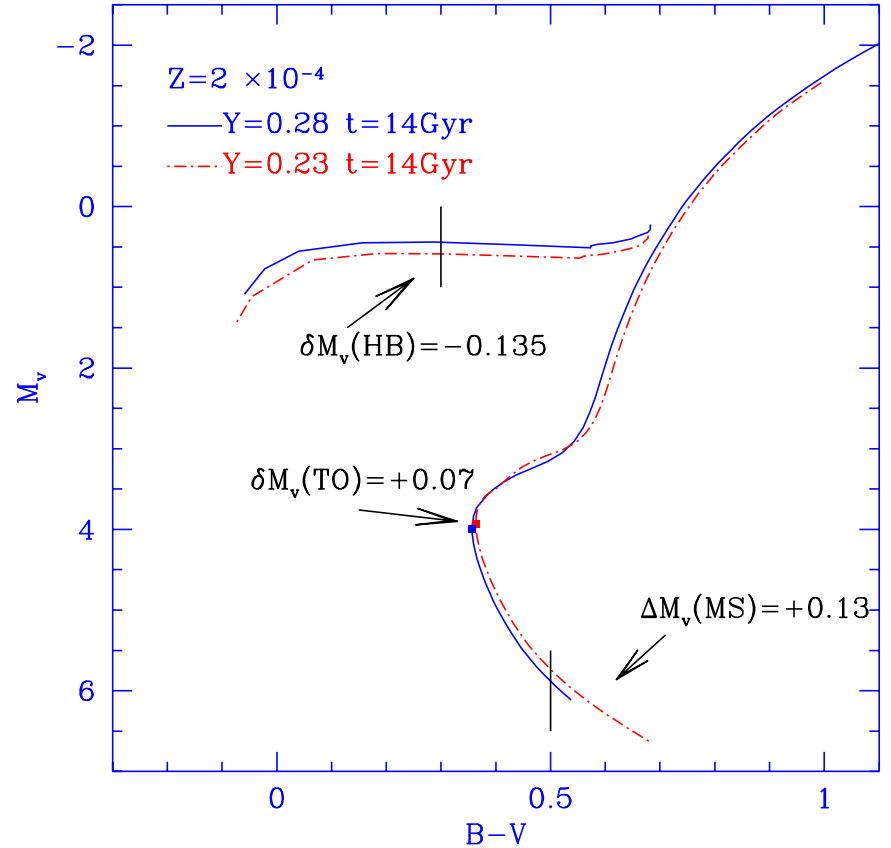

Fig. 1. Comparison of two isochrones of age $14 \mathrm{Gyr}, Z=2 \times 10^{-4}$ and different helium abundance. The ZAHBs for the two $Y$ values are also shown, down to a minimum HB mass of $M=0.6 M_{\odot}$.

expelled at low velocity by the stellar bodies during the AGB evolution, various consequences can arise. Given the depth of the potential well, a significant fraction of this material is not lost by a globular cluster, unless there is a significant interaction with external systems (e.g. another cluster, or a giant molecular cloud, or the galactic bulge). New stars can form out of this matter, or pre-existing diffuse material can be polluted, or low mass unevolved stars can be polluted to various degrees.

In order to evaluate the impact of a possible population having a different chemical composition on the color magnitude diagram of a globular cluster, in this article we compare isochrones having primordial helium abundance ${ }^{2}$, which we set at $Y=0.23$ for $Z=2 \times 10^{-4}$, and at $Y=0.24$ for $Z=10^{-3}$, with models having a main sequence enhanced helium $Y=0.28$. We compute models for all evolutionary phases and compare the location of the main sequence (MS), turnoff (TO), red giants (RGB) and horizontal branch (HB). The lower main sequence models, and the detailed evolution along the RGB are considered in a forthcoming paper (Montalbán et al. 2002). In Figs. 1 and 2, the low mass cutoff of each main sequence is $0.6 M_{\odot}$. Notice that the $Y=0.28,0.6 M_{\odot}$ models are much more luminous (by more than $0.4 \mathrm{mag}$ ) than the standard helium models. Nevertheless, the relative locations of the standard and enhanced helium isochrones show that it is not

\footnotetext{
${ }^{2}$ We take $Y=0.24$ as standard value for the metallicity $Z=10^{-3}$. Implicitly, we are assuming that the Big Bang value was $Y=0.23$, and that there is a small increase in $Y$ for the matter reaching a metallicity $Z=10^{-3}$. Alternatively, we may regard $Y=0.23$ and $Y=0.24$ both as values indicative of the helium abundance emerging from the Big Bang. The value $Y=0.228 \pm 0.005$ was derived by Pagel et al. (1992), whereas today a larger value, $Y=0.244 \pm 0.002$ results from Izotov $\&$ Thuan (1998) analysis of the helium emission lines in HII regions of metal poor dwarf galaxies.
}

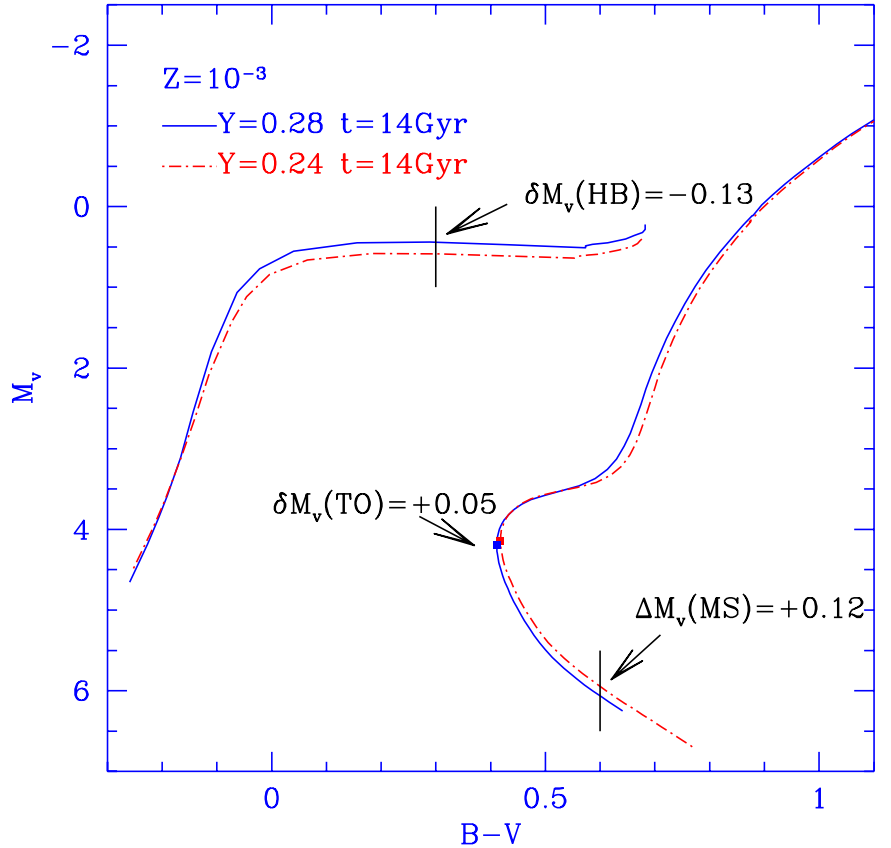

Fig. 2. The same comparison as in Fig. 1 , for $Z=10^{-3}$. In this case, we have extended the ZAHB down to masses as small as $M=0.5 M_{\odot}$ for $Y=0.24$ and $M=0.491 M_{\odot}$ for $Y=0.28$. These additional (bluer) models below $0.6 M_{\odot}$ are necessary for the simulations presented in Sect. 2.1.

obvious how to discriminate between a population with uniform helium content and one with a spread in helium, for what concerns TO and RGB evolution. On the other hand, the morphology of the HB is affected by a helium variation, directly through the difference in the TO mass for a given age and we argue that a helium variation may be the main reason for the occurrence of extreme blue tails in clusters such as NGC 6752 and $\mathrm{M} 13$.

This conclusion differs from the common hypothesis put forward in recent years: a helium enrichment in the envelope as a consequence of deep mixing in today evolving red giants has in fact been suggested as a way to obtain blue and very blue HBs (Langer \& Hoffman 1995; Sweigart 1997). As a matter of fact, an increased helium abundance can help in reaching a HB position bluer than the RR Lyrae variables (at the expense of a more or less substantial increase in luminosity), but the extremely blue locations always require an extreme mass loss, because the envelope mass has to be in any case smaller than $0.01 M_{\odot}$ (see the discussion in Caloi 2001). In addition, Weiss et al. (2000) have shown that the helium enhancement needed to explain the exclusively blue HB morphology would produce related chemical inhomogeneities in red giants much stronger than observed. So an envelope helium content larger than the cosmological one does not help in obtaning the structures which make up the long blue tails in, e.g., M 13 and NGC 6752. We show that the situation is different if helium is larger in the whole stellar structure, that is, if cluster stars form out of material with enhanced helium. We considered this hypothesis at the light of the present state of the art in stellar modelling. 
Table 1. Input data for the HB models.

\begin{tabular}{lcll}
\hline \hline$Y$ & $Z$ & $Y_{\mathrm{e}}$ & $M_{\mathrm{c}} / M_{\odot}$ \\
\hline 0.23 & $2 \times 10^{-4}$ & 0.235 & 0.508 \\
0.28 & $2 \times 10^{-4}$ & 0.285 & 0.497 \\
0.24 & $10^{-3}$ & 0.248 & 0.497 \\
0.28 & $10^{-3}$ & 0.2875 & 0.489 \\
\hline
\end{tabular}

\section{The models and the comparison with observations}

Appropriate evolutionary models have been computed with the code ATON2.0, described in Ventura et al. (1998) and Mazzitelli et al. (1999). We considered models with $Y=0.28$, and two values for $Z: 2 \times 10^{-4}$ and $10^{-3}$, to be compared with tracks with $Y=0.23, Z=2 \times 10^{-4}$, and $Y=0.24, Z=10^{-3}$. The increase in the helium content has been taken meaningful, but not such as to a priori alter dramatically the currently accepted evolution of a GC low mass star, and it roughly agrees with the expected helium enhancement in the ejecta of massive, metal poor AGB stars. We have built isochrones for GC ages, and transformed the luminosity and $T_{\text {eff's into observa- }}$ tional magnitudes and colors by using the transformations by Castelli et al. (1997) ${ }^{3}$.

Figures 1 and 2 show the location of the 14 Gyr isochrones in the HR diagram, and the relevant variations in the main loci. Similar variations hold for different ages.

The computed isochrones show that there is a small age difference for the same TO magnitude $(\sim 0.8 \mathrm{Gyr}$ for $Z=10^{-3}$, and $\sim 1 \mathrm{Gyr}$ for $Z=2 \times 10^{-4}$ respectively, the larger helium the younger), while for the same age one finds 0.05 (0.07) mag difference (the larger helium the fainter, see Figs. 1 and 2). The main sequence location for the higher helium is $0.12(0.13)$ mag fainter and the helium core mass at the red giant tip is $0.008 M_{\odot}$ smaller for the higher helium tracks $\left(M_{\text {core }}=0.497\right.$ and $0.489 M_{\odot}$, respectively, for $Z=10^{-3}$ ). The color difference between the low and high helium isochrones is $\lesssim 0.015 \mathrm{mag}$ both along the RGB and $\simeq 1$ mag below the MS TO. In a cluster with a dispersion in helium abundance, such a small difference is certainly beyond present detection capabilities. A small population of binaries would make even more difficult to distinguish a possible broadening of the mean loci.

\subsection{The horizontal branch}

Table 1 shows the input parameters for the HB models, namely the helium core mass $M_{\mathrm{c}}$ and the surface helium abundance $Y_{\mathrm{e}}$. $M_{\mathrm{c}}$ is the helium core mass at the RG tip, appropriate for ages between 10 and $16 \mathrm{Gyr}$, according to the present models. $Y_{\mathrm{e}}$ is increased with respect to the initial value, due to the first dredge up. The zero age horizontal branch (ZAHB) loci are shown in

\footnotetext{
3 Tables of isochrones and HB models in $M_{\mathrm{v}}, B-V$ and $V-I$ are available on request. We are working to make available the models also in other (HST) passbands, as well as a computation tool to build HB synthetic populations, taking into account helium content spreads.
}

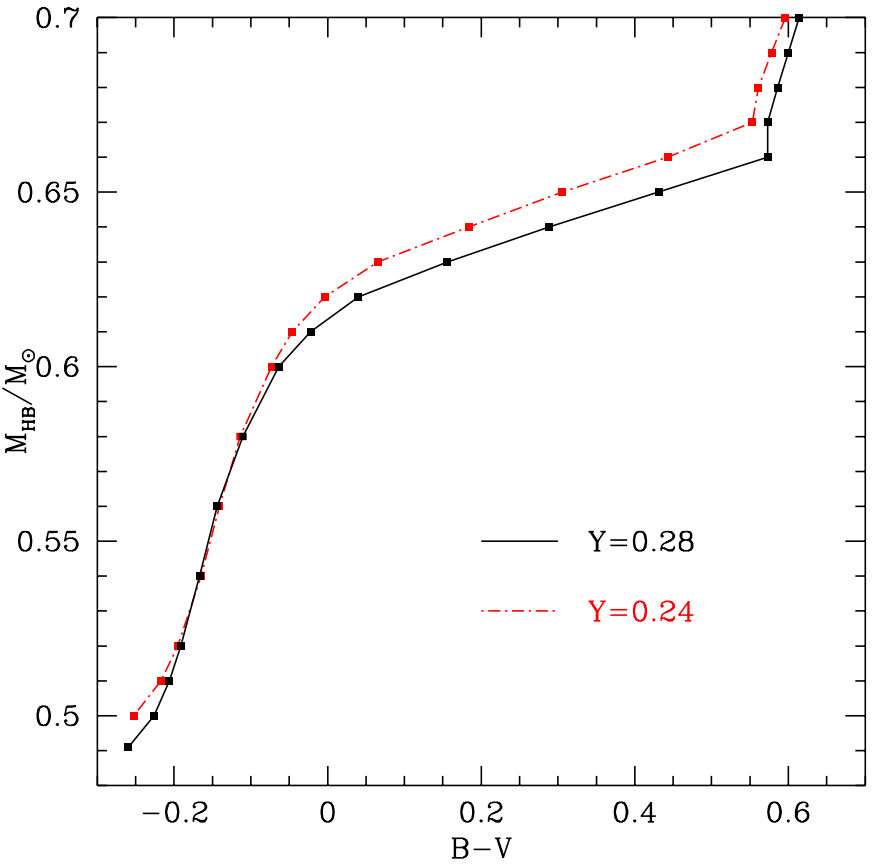

Fig. 3. The mass versus $B-V$ distribution in ZAHB for the two helium contents in the $Z=10^{-3}$ case. Formally, the $Y=0.28$ models are redder that the $Y=0.24$ models, for the same total mass, except for the smaller masses. However, the mass loss mechanism at the red giant branch operates on a smaller total mass for the high helium case, so that it is easier to achieve a smaller remnant mass and bluer stars.

Figs. 1 and 2, while Fig. 3 shows the HB mass versus the ZAHB $B-V$ for the $Z=10^{-3}$ case.

For what concerns the actual distribution along the HB locus, the relevant feature is that the isochrone with initial helium content of $Y=0.28$ reaches the giant tip with a mass smaller of $0.05 M_{\odot}$ than in the case with standard helium. This is a well known result of the evolution - see, e.g., Iben \& Renzini (1984) - which in our case provides an important clue to the HB morphology of those globular clusters in which we may suspect that there is a spread or a dichotomy in the helium abundance. Although Fig. 3 shows that, at a given $B-V$ color, the $Y=0.28$ ZAHB mass is smaller by $\simeq 0.01 M_{\odot}$ than the mass for lower helium, we expect that the helium enriched stars will occupy bluer HB loci, mainly due to the difference in the evolving total mass.

In order to make this point more clear, we computed a few simulations of the HB distributions expected for different assumptions on mass loss and helium content of the sample stars. These simulations are not intended to cover in an exhaustive way all possible cases, but simply to exemplify the main new point of the present work. We make the assumption that the evolving giants include two components: the first one has initial abundance of $Z=0.001$ and $Y=0.24$; the second one has the same metal abundance, but a uniform distribution of the initial helium content between $Y=0.24$ and $Y=0.28$. We also ran other simulations testing the hypothesis of a gap in the helium content distribution between the two components. This would be the case if a new generation of stars were born in the cluster directly from the wind ejecta of the massive AGBs. 


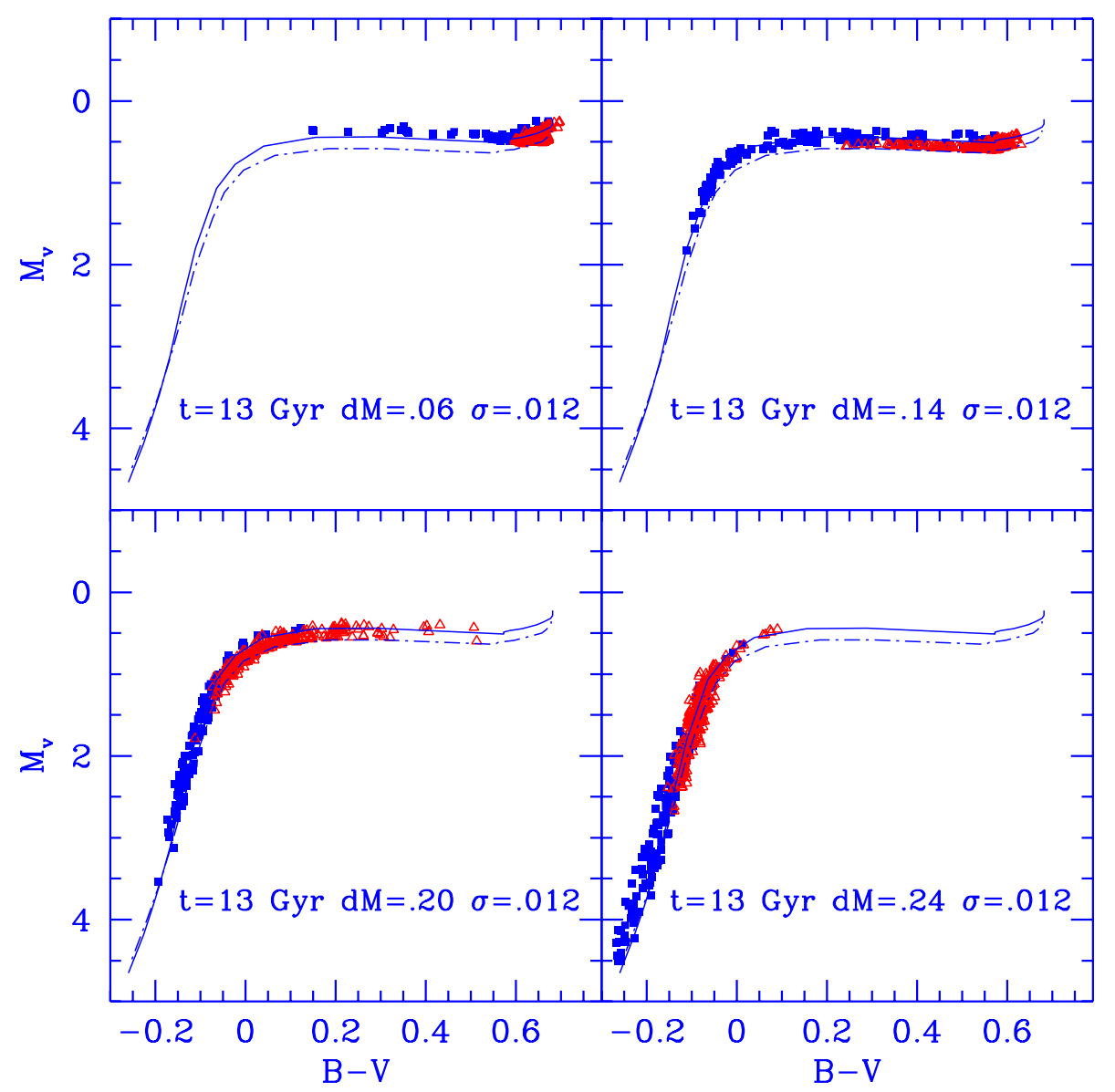

Fig. 4. The four panels show the synthetic HB for different choices of the average mass loss (d $M$ in $\left.M_{\odot}\right)$, and dispersion in mass loss $\left(\sigma\right.$, in $\left.M_{\odot}\right)$. The age assumed is $13 \mathrm{Gyr}$ and the metallicity is $Z=10^{-3}$. The simulations contain 400 stars, of which 250 have $Y=0.24$ (triangles) and the remaining 150 have $Y$ randomly chosen between 0.24 and 0.28 (squares).

We assume that the individual $\mathrm{HB}$ masses are the result of a mass loss process which goes on along the RGB, with a Reimers' (1997) like mass loss law. We assume that the average mass lost in this process is $\delta \mathrm{M}$, with a Gaussian distribution having mass dispersion $\sigma$ (in this way, stars with identical chemical compositions may populate different part of the ZAHBs). Further, as Reimers' mass loss rate increases with decreasing $M_{\mathrm{RG}}$ (see the discussion in Lee et al. 1994), we also take into account that more mass is lost on average from the helium rich (and smaller mass) evolving giants. We ran various simulations with different values of $\delta M$ and $\sigma^{4}$, and computed the distribution of stars along the HB for several ages. Here we show a few examples of the results obtained for an age of 13 Gyr. Very similar results can be obtained for larger (smaller) ages, by decreasing (increasing) accordingly the average mass loss. The total number of HB stars originated from main sequence stars with $Y=0.24$ is taken to be 250 . To these, we add from 100 to 150 stars deriving from main sequence structures with helium content varying from 0.24 to 0.28 , as mentioned before.

In the four panels of Fig. 4, the HB members with original $Y=0.24$ are indicated by open triangles, while squares

\footnotetext{
${ }^{4}$ We do not assume any explicit dependence of mass loss on the metallicity, as we are only giving examples of the resulting distributions for one metallicity only.
}

indicate stars deriving from the He-rich population described above (original $Y$ from 0.24 to 0.28 ); evolution out of the ZAHB is taken into account, together with a small spread in $V\left(\sigma_{v}=0.005\right)$ and in $B-V\left(\sigma_{B-V}=0.01\right)$, which again simulate the impact of observational errors. Cluster members with low helium abundance give origin to the bulk of HB distribution, while, as expected, the high helium structures are able to populate much bluer HB regions. This is in part a consequence of the smaller mass evolving at the TO (up to $0.05 M_{\odot}$ for the stars with $Y=0.28$ ), and in part because of the extra mass loss due to the smaller mass of the evolving giants (up to $0.03 M_{\odot}$ for $Y=0.28$; see Lee et al. 1994).

When the average mass loss is small, most stars would evolve on the red side of the HB (top panels of Fig. 4). However, stars on the red HB and within the RR Lyrae strip computed with a distribution of initial values of the helium abundance would be on average more luminous and yield a substantially thicker distribution than expected in the standard (low helium) case. For clusters more metal rich than considered here, in which the HB is restricted to the red of the RR Lyrae gap, this feature has probably been observed: in 47 Tuc (Briley 1997), CN-strong HB stars appear on the average slightly more luminous ( $0.05 \mathrm{mag}$ ) and tend to be bluer, than their $\mathrm{CN}$-weak counterparts. By increasing the average mass loss in our simulations, (top right panel in Fig. 4) the helium rich stars begin to 


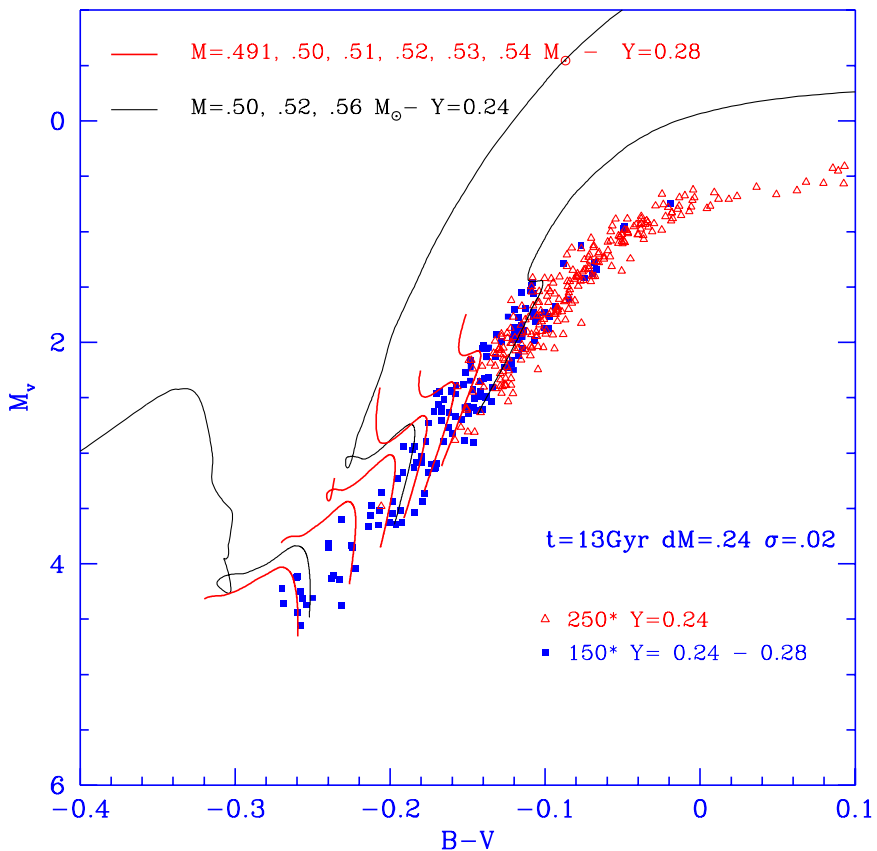

Fig. 5. A simulation is shown together with the corresponding evolutionary tracks.

populate the whole HB extension from blue to red, even assuming a relatively small dispersion in the mass loss of $\sigma=0.012$.

If the HB is of the type shown in the bottom left panel of Fig. 4, the luminosity level of the HB is determined by the $Y=0.24$ stars, so that the problem of predicting too high a luminosity for the HB if there is a helium spread is not relevant: all the helium rich stars, in fact, are confined to the blue side of the HB, due to their smaller mass and larger mass loss in the RGB phase.

In exclusively blue HBs (bottom right of Fig. 4), again the higher helium structures are confined on the blue tail, where helium abundance does not affect noticeably ZAHB position and early evolution (see, e.g., Caloi 2001).

In Fig. 5 we show the typical HB distribution in a simulation including 250 stars with $Y=0.24$ and 150 stars with $Y$ uniformely distributed between 0.24 and 0.28 . The corresponding tracks are also shown. The chosen mass loss rate is such that only the helium rich stars populate the extreme blue tail. Other experiments with different values of the mean mass loss and mass dispersion, are shown in Figs. 6 and 7. Here we have also introduced a gap in the helium range of the enriched population ( $Y$ from 0.26 to 0.28 in Fig. 6 and a fixed $Y=0.28$ for Fig. 7). In Figs. 6 and 7 we also show the histogram of the luminosity function of HB stars. We see that a dip in the distribution appears at the magnitude corresponding to the change in the helium abundance, and we consider this to be a meaningful feature. Other features at larger $M_{\mathrm{v}}$ do not appear to be statistically significant.

With values for the parameters largely in the accepted range, we can obtain the morphologies of well known globular clusters with blue tails (cf. Piotto et al. 1999; Buonanno et al. 1986; Ferraro et al. 1998). A very blue clump well separated from a "normal" blue HB (that is, derived from giants

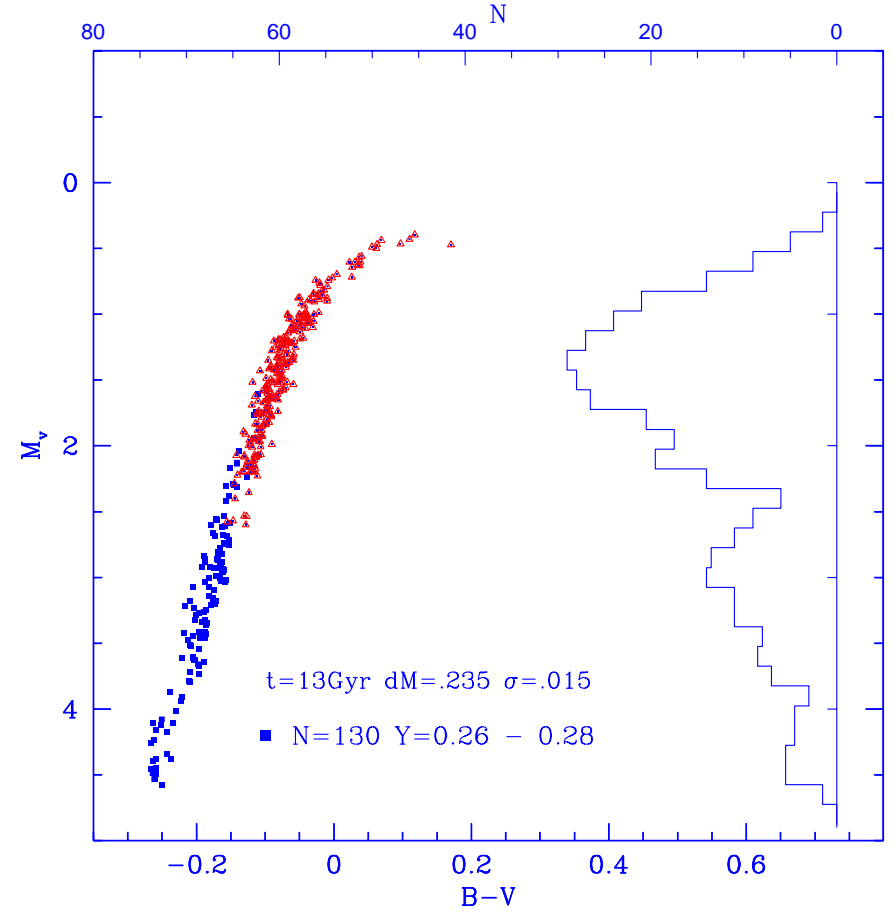

Fig. 6. Simulation containing 380 stars, 250 of which have $Y=0.24$, and 130 helium randomly chosen between 0.26 and 0.28 .

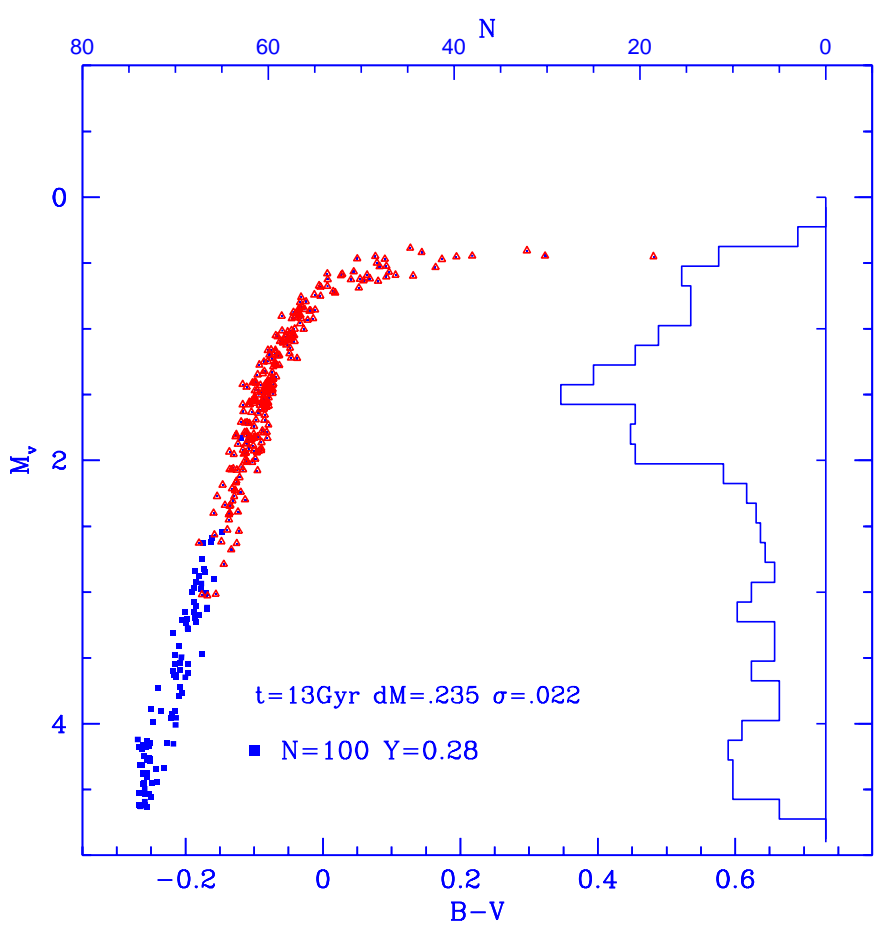

Fig. 7. Simulation containing 350 stars, 250 of which have $Y=0.24$, and 100 have $Y=0.28$.

with standard helium) can be obtained if helium in the enriched population peaks at $Y=0.28$ (Fig. 7). The bluest HB structures have a hydrogen rich envelope of the order of $0.001 M_{\odot}$, and evolve vertically in the CM diagram at a constant rate (as predicted by all evolutionary computations), partially filling the gap. 


\section{Conclusion}

The problem of chemical inhomogeneities in GCs is part of the wider question of their chemical history, which appears more and more complex, and differing from cluster to cluster. As put in evidence, e.g., by Sneden (2000), the neutron-capture elements $\mathrm{Ba}$ and $\mathrm{Eu}$ have variable abundances without obvious connection to overall cluster metallicity. Besides, variations in $\mathrm{Si}$ content are also observed from cluster to cluster.

The fact that field stars do not show evidence of the substantial abundance re-shuffling between $\mathrm{CNO}$ elements and $\mathrm{Na}$, $\mathrm{Mg}$ and $\mathrm{Al}$ strongly suggests some intra cluster processes at the origin of the phenomenon. Here we examine one possible aspect of these (admittedly) rather vague processes.

We discussed the evolutionary properties of GC stars in which variations of the primordial helium content are allowed. These variations are attributed to self-pollution by an initial population of intermediate mass stars, and are expected on the basis of the same stellar models which are thought to produce the $\mathrm{CNO}$ variations among members of several GCs.

The observation of chemical peculiarities (dichotomy in the strength of $\mathrm{CN}$ lines, $\mathrm{Na}$ and $\mathrm{Al}$ enhancement) in some $\mathrm{GC}$ giants suggested already a long time ago (Cottrell \& Da Costa 1981; Norris et al. 1981) that intermediate mass stars may have played a role in creating some inhomogeneity in the primordial cloud of certain GCs. In particular, Norris et al. (1981) put in relation chemical peculiarities with blue tails (NGC 6752), and suggested precisely the mechanism which is at the basis of the present investigation.

We find that a spread in the helium content does not affect the morphology of the MS, TO and RGB in an easily observable way. The simulations in Figs. 4-7 show that also the HB luminosity level is affected in a limited way, since only an RR Lyrae region populated exclusively by structures with the maximum $Y$ allowed (0.28) would stand out clearly as peculiarly luminous. On the other hand, such an occurrence should not be verified with standard values for the mean mass loss ( $\Delta M \gtrsim 0.2 M_{\odot}$, see Lee et al. 1994).

So age determination should not be affected in a substantial way. There may be a small effect if we use as distance indicator the subdwarf main sequence, since it is reasonable to assume that subdwarfs have a helium content not affected by self-pollution.

On the other hand, the helium spread may constitute part of the "second parameter" problem. The presence of very blue extensions in many clusters with predominantly blue $\mathrm{HB}$, and sometimes also in HBs with RR Lyrae variables and red members - NGC 2808 (Walker 1999), M 62 (Caloi et al. 1987; Brocato et al. 1996) - may be related to the second parameter problem, but this side of the question will not be considered here. The extremely small envelope masses necessary to populate these blue tails require mass losses, and/or ages, much larger than the average values required to explain also exclusively, but not extended, blue HBs, in case of uniform cosmological helium abundance. As we saw before, the smaller TO masses (for a given age) of a helium enriched structure are crucial to obtain the tiny envelopes of very blue HB stars.
The difficulties with the present hypothesis are mainly due to the enrichment scenario, since it is not easy to pollute a large fraction of original intracluster matter in a substantial way (Cannon et al. 1998). We note that the requirement of the pollution of a large fraction of the cluster population comes from the roughly bimodal distribution of $\mathrm{CN}$ abundances and from the well developed blue tails in many clusters, if they are related to the helium enhancement phenomenon (M 13, NGC 6752).

In the self-enrichment scenarios, if the extreme blue tails can indeed be attributed to the difference among the total masses of the stars evolving in the RGB, due to their different helium content, the helium content must be larger also in the center of the stars, to affect their hydrogen burning lifetimes, and therefore we should conclude that the self-pollution has occurred either on the gas from which the helium rich stellar generation was formed, or it has occurred during the phases of the stars evolution in which they were fully convective. It is also possible that the ejecta of AGBs, collected at the center of the cluster, directly form other stellar generations. If this is the case, some gaps in the HB stellar distributions could reflect discontinuities in the helium content of the constituent matter, due to the difference in the helium abundance in the ejecta of AGBs of different mass (Ventura et al. 2002) and to some peculiar modalities of star formation from these ejecta.

Acknowledgements. This work is supported by the Italian Ministry of University, Scientific Research and Technology (MUIR) within the Cofin 1999 Project: "Stellar Dynamics and Stellar Evolution in Globular Clusters: a Challenge for the New Astronomical Instruments" and the Cofin 2002 Project: "New frontiers in the study of pulsars".

\section{References}

Bell, R. A., Cannon, R. D., Harris, G. L. H., \& Hesser, J. E. 1981, ApJ, 249, 637.

Briley, M. M. 1997, AJ, 114, 1051

Brocato, E., Buonanno, R., Malakhova, Y., \& Piersimoni, A. M. 1996, A\&A, 311, 778

Buonanno, R., Caloi, V., Castellani, V., et al. 1986, A\&AS, 66, 79

Caloi, V., Castellani, V., \& Piccolo, F. 1987, A\&AS, 67, 181

Caloi, V. 2001, A\&A, 366, 91

Cannon, R. D., Croke, B. F. W., Bell, R. A., Hesser, J. E., \& Stathakis, R. A. 1998, MNRAS, 298, 601.

Castelli, F., Gratton, R. G., \& Kurucz, R. L. 1997, A\&A, 318, 841

Catelan, M., \& de Freitas Pacheco, J. A. 1995, A\&A, 297, 345.

Cottrell, P. L., \& Da Costa, G. S. 1981, ApJ, 245, L79

Da Costa, G. S., \& Cottrell, P. L. 1980, ApJ, 236, L83

D'Antona, F., Gratton, R., \& Chieffi, A. 1983, Mem. S. A. It., 54, 173

Ferraro, F. R., Paltrinieri, B., Pecci, F. F., Rood, R. T., \& Dorman, B. 1998, ApJ, 500, 311

Gratton, R. G., Bonifacio, P., Bragaglia, A., et al. 2001, A\&A, 369, 87

Iben, I., \& Renzini, A. 1984, Phys. Rep., 105, 329

Izotov, Y. I., \& Thuan, T. X. 1998, ApJ, 500, 188

Kraft, R. P. 1994, PASP, 106, 553

Kraft, R. P., Sneden, C., Smith, G. H., et al. 1997, AJ, 113, 279

Langer, G. E., \& Hoffman, R. D. 1995, PASP, 107, 1177

Lee, Y. W., Demarque, P., \& Zinn, R. 1994, ApJ, 423, 248

Mazzitelli, I., D’Antona, F., \& Ventura, P. 1999, A\&A, 348, 846

Montalbán, J., Ventura, P., \& D’Antona, F. 2002, in preparation 
Norris, J., Cottrell, P. L., Freeman, K. C., \& Da Costa, G. S. 1981, Sweigart, A. V. 1997, ApJ, 474, L23 ApJ, 244, 205

Thoul, A., Jorissen, A., Goriely, S., et al. 2002, A\&A, 383, 491

Pagel, B. E. J., Simonson, E. A., Terlevich, R. J., \& Edmunds, M. G. Ventura, P., Zeppieri, A., Mazzitelli, I., \& D’Antona, F. 1998, A\&A, 1992, MNRAS, 255, 325 334, 953

Pilachowski, C. A., Sneden, C., Kraft, R. P., \& Langer, G. E. 1996, AJ, 112, 545

Piotto, G., Zoccali, M., King, I. R., et al. 1999, AJ, 118, 1727

Reimers, D. 1977, A\&A, 57, 395

Smith, G. H., \& Norris, J. E. 1993, AJ, 105, 173

Sneden, C. 2000, in The Galactic Halo: From Globular Cluster to Field entura, P., D’Antona, F., Mazzitelli, I., \& Gratton, R. 2001, ApJ, 550, L65

Ventura, P., D’Antona, F., \& Mazzitelli, I. 2002, A\&A, 393, 215

Walker, A. R. 1999, AJ, 118, 432

Weiss, A., Denissenkov, P. A., \& Charbonnel, C. 2000, A\&A, 356, 181

Stars, ed. A. Noels, P. Magnain, \& D. Caeo, 35th Liege Int. Astr. Coll., 159 